\title{
CARACTERIZACIÓN ESPACIAL DE LA POBLACIÓN MIGRANTE EN LA ZONA FRONTERIZA DE LA PROVINCIA DE SUCUMBÍOS
}

\author{
Mayo. De E. Marcelo Torres \\ Curso de Estado Mayor
}

\begin{abstract}
RESUMEN
La migración forzosa internacional es causa y efecto de diversas formas de conflicto al interior de un Estado o entre estos. Hay una crítica tendencia de crecimiento sostenido de movimientos simultáneos de masas poblacionales en distintas regiones del mundo y Ecuador no está aislado de esta realidad. Los problemas internos de Colombia y Venezuela ocasionan el ingreso y permanencia de migrantes irregulares con altos niveles de vulnerabilidad socio-económica y necesidades de atención emergente en nuestro país. Por otro lado, los problemas relacionados a las amenazas como el narcotráfico, los cultivos ilícitos y los grupos irregulares armados en los Departamentos colombianos de Nariño y Putumayo, influyen en el incremento del nivel delictivo y la reducción de la seguridad colectiva en la provincia ecuatoriana de Sucumbíos. Ante este escenario y por la necesidad de recursos económicos, la población migrante podría verse inmersa en actividades ilegales. Las Fuerzas Armadas son responsables del control en la Zona Fronteriza y por lo tanto deben implementar todas las capacidades requeridas para garantizar la seguridad en la misma. La caracterización de la población migrante es indispensable para la generación de inteligencia útil para la planificación de operaciones en el ámbito interno. El uso de sistemas de información geográfica e infraestructuras de datos espaciales son herramientas ideales para este propósito, a través de las cuales se brindará apoyo para la efectiva y oportuna toma de decisiones en el Comando de Operaciones No. 1 "NORTE".
\end{abstract}

Palabras Claves: migración irregular, seguridad colectiva, caracterización espacial, operaciones en el ámbito interno. 


\begin{abstract}
Forced international migration is the cause and effect of various forms of conflict within or between a State. There is a critical trend of sustained growth of simultaneous movements of population masses in different regions of the world and Ecuador is not isolated from this reality. The internal problems of Colombia and Venezuela cause the entry and permanence of irregular migrants with high levels of socio-economic vulnerability and needs for emergent attention in our country. On the other hand, the problems related to threats such as drug trafficking, illicit crops and irregular armed groups in the Colombian Departments of Nariño and Putumayo, influence the increase in the level of crime and the reduction of collective security in the province of Sucumbíos. Given this scenario and the need for economic resources, the migrant population could be immersed in illegal activities. The Armed Forces are responsible for control in the Border Zone and therefore must implement all the capabilities required to ensure security in it. The characterization of the migrant population is fundamental for the generation of useful intelligence for the planning of military operations in the internal sphere. The use of geographic information systems and spatial data infrastructures are ideal tools for this purpose, through which support will be given to timely and effective decision-making of Comando de Operaciones No. 1 "NORTE".
\end{abstract}

Keywords: irregular migration, collective security, spatial characterization, operations in the internal sphere

\section{Introducción}

A nivel global, las crecientes desigualdades de riqueza, los altos índices de inseguridad, las presiones políticas, ambientales, religiosas, étnicas y demográficas, entre otras, obligan continuamente a millones de habitantes a buscar refugio fuera de sus propios países. Según Martínez (2005), la migración internacional es causa y efecto de diversas formas de conflicto, evidenciando deficiencias profundas en la concepción e implementación del desarrollo sostenible como una herramienta eficaz de gobierno en cada uno de los Estados de origen.

Una evidencia de ello es la crítica tendencia de crecimiento sostenido de movimientos simultáneos de masas poblacionales en distintas regiones del mundo, ocasionando la denominada "globalización de la migración" (Castles y Miller, 2004). Este concepto incluye a un amplio grupo de países receptores de una variada gama de ciudadanos migrantes con diversos antecedentes económicos, sociales y culturales, provocando una combinación infinita de identidades, mestizajes y mutaciones sociales. Esta diversidad incrementa la complejidad del análisis al cual se someten quienes administran las políticas migratorias nacionales, con la finalidad de tomar decisiones para gestionar el fenómeno inmigratorio en sus territorios.

En cuanto a América Latina, el Alto Comisionado de las Naciones Unidas para los Refugiados (ACNUR) y la Organización Internacional para las Migraciones (OIM), continuamente convocan a la comunidad internacional a brindar mayor apoyo a los países y a las comunidades que en la región están recibiendo cantidades crecientes de refugiados y migrantes de países como Venezuela, Colombia, Cuba y Haití.
En efecto, y a fin de tener una idea, solo en el caso de Venezuela, un estimado de 2’300.000 ciudadanos están viviendo en el extranjero, de los cuales más de 1'600.000 abandonaron su país desde el año 2015. El 90\% de ellos fueron a países suramericanos (Organización Internacional para las Migraciones de las Naciones Unidas, 2018).

\section{Migración hacia Ecuador: orígenes y estadísticas}

Ecuador se presenta como uno de los principales países receptores en la región debido a la a la presencia del dólar como su moneda oficial y a la apertura y flexibilidad de sus políticas migratorias, entre otros factores (Zurita, González, y Dávalos, 2017).

En efecto y, en relación a la migración venezolana hacia nuestro país, la tabla 1 refleja el flujo de ciudadanos provenientes de este país, entre los años 2013 y 2017 (Ministerio del Interior, 2018). Se mencionan únicamente a los registros logrados por las Unidades de Control Migratorio (UCM) conformadas por puertos, aeropuertos $\mathrm{y}$ pasos fronterizos legales a nivel nacional, sin incluir los datos de quienes entraron o salieron por pasos fronterizos ilegales.

En la figura 1 se observa gráficamente los datos detallados anteriormente:

\begin{tabular}{c|c|c|c|c|c|c|c|c|c}
\multicolumn{2}{c|}{2013} & \multicolumn{2}{c|}{2014} & \multicolumn{2}{c|}{2015} & \multicolumn{2}{c|}{2016} & \multicolumn{2}{c}{2017} \\
\hline Arribo & Salida & Arribo & Salida & Arribo & Salida & Arribo & Salida & Arribo & Salida \\
\hline 99984 & 99106 & 120154 & 116530 & 94715 & 89751 & 102369 & 78696 & 288005 & 226867 \\
\hline
\end{tabular}

Tabla 1. Flujo de ciudadanos venezolanos por las UCM en el Ecuador (2013-2017)

Fuente: Ministerio del Interior, 2018 


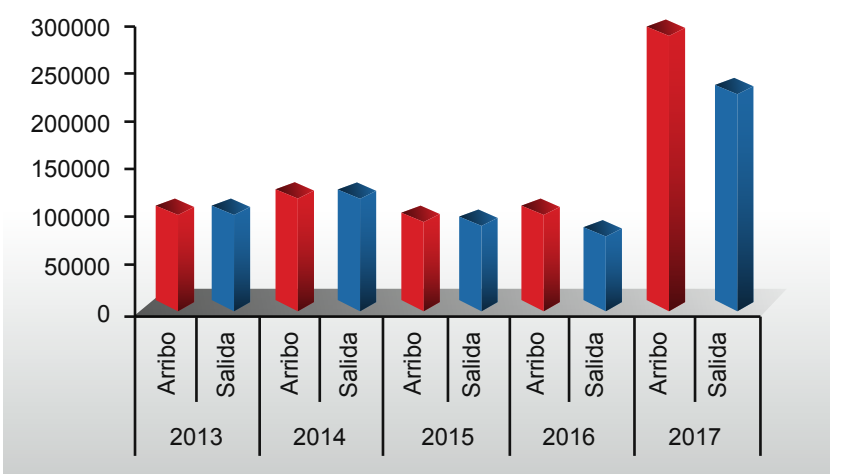

Figura 1. Relación entre arribo y salida de venezolanos a través de las UCM de Ecuador (2013 y 2017)

Fuente: Ministerio del Interior, 2018, adaptado por el autor.

Como se puede observar, el año 2017 cuenta con el mayor flujo de venezolanos registrado en el periodo referido, lo cual coincide con el año en que iniciaron los amplios movimientos migratorios de la población de este país a diferentes lugares de la región.

Además, en cada uno de los años analizados, es posible determinar que los arribos superan a las salidas, lo cual permite concluir que la población migrante venezolana que se erradicó en Ecuador, se incrementó continuamente. Ello se detalla en la tabla 2:

\begin{tabular}{l|c|c|c|c|c|c|c|c|c|c} 
& \multicolumn{2}{|c}{$\mathbf{2 0 1 3}$} & \multicolumn{2}{c}{$\mathbf{2 0 1 4}$} & \multicolumn{2}{c}{$\mathbf{2 0 1 5}$} & $\mathbf{2 0 1 6}$ & \multicolumn{2}{c}{$\mathbf{2 0 1 7}$} \\
& Arribo & Salida & Arribo & Salida & Arribo & Salida & Arribo & Salida & Arribo & Salida \\
\cline { 2 - 9 } & $\mathbf{9 9 9 8 4}$ & $\mathbf{9 9 1 0 6}$ & $\mathbf{1 2 0 1 5 4}$ & $\mathbf{1 1 6 5 3 0}$ & $\mathbf{9 4 7 1 5}$ & $\mathbf{8 9 7 5 1}$ & $\mathbf{1 0 2 3 6 9}$ & $\mathbf{7 8 6 9 6}$ & $\mathbf{2 8 8 0 0 5}$ & $\mathbf{2 2 6 8 6 7}$ \\
\hline Población que se quedó en el país por año & 878 & 3624 & 4964 & 23673 & 61138 \\
\hline $\begin{array}{l}\text { Población que se quedó en el país por año } \\
\text { (porcentaje) }\end{array}$ & $0.88 \%$ & \multicolumn{2}{|c|}{$3.02 \%$} & \multicolumn{2}{|c|}{$5.24 \%$} & $23.13 \%$ & $21.23 \%$ \\
\hline Incremento acumulado anual & \multicolumn{2}{|c|}{878} & 4502 & 9466 & 33139 & 94277 \\
\hline
\end{tabular}

Tabla 2. Detalle de ciudadanos venezolanos que arribaron y se quedaron en Ecuador entre 2013-2017

Fuente: Ministerio del Interior, 2018, adaptado por el autor.

Es posible inferir que hasta el 31 de diciembre de 2017, 94.277 ciudadanos venezolanos se quedaron en el país luego de su ingreso por las UCM (asumiendo que los mismos no han emigrado a otros países a través de pasos fronterizos no oficiales).

En la figura 2 se observa gráficamente el incremento acumulado anual de población que se quedó en el país entre 2013 y 2017:

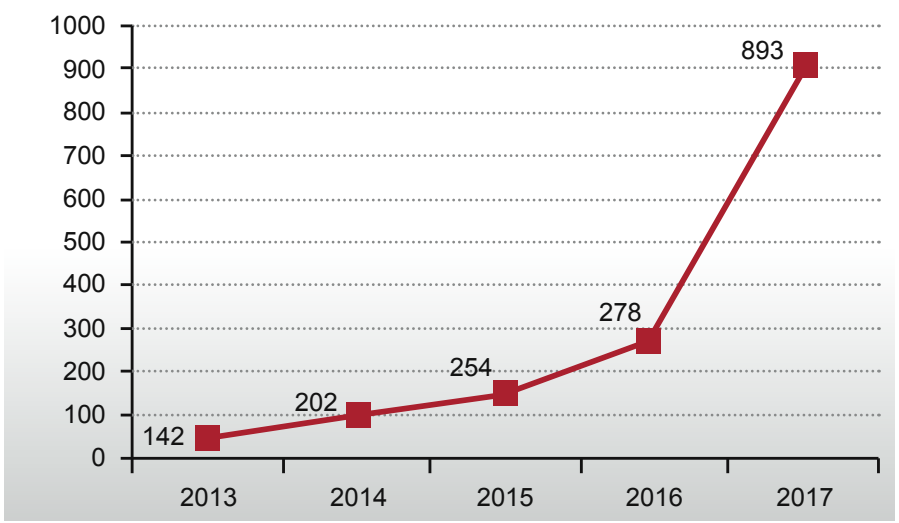

Figura 2. Incremento acumulado anual de población venezolana que se quedó en Ecuador (2013 - 2017) Fuente: Ministerio del Interior, 2018, adaptado por el autor. 
Complementariamente, y según el Ministerio del Interior (2018), este incremento continuó en el transcurso del año 2018. Así, hasta el mes de septiembre de dicho año, se registró un total de 135.000 venezolanos que permanecen en el territorio nacional.

En cuanto respecta a la provincia de Sucumbíos, objeto de este estudio, en el ingreso y salida por el Paso Fronterizo (PF) de Lago Agrio, existe una relación similar entre ingresos y salidas, tal como se observa en la tabla 3 y figura 3:

\begin{tabular}{|c|c|c|c|c|c|c|c|c|c|c|}
\hline & \multicolumn{2}{|c|}{2013} & \multicolumn{2}{|c|}{2014} & \multicolumn{2}{|c|}{2015} & \multicolumn{2}{|c|}{2016} & \multicolumn{2}{|c|}{2017} \\
\hline & Arribo & Salida & Arribo & Salida & Arribo & Salida & Arribo & Salida & Arribo & Salida \\
\hline & 648 & 506 & 256 & 196 & 219 & 167 & 107 & 83 & 863 & 248 \\
\hline $\begin{array}{l}\text { Población que cruzó el paso fronterizo } \\
\text { Lago Agrio (Sucumbíos) }\end{array}$ & \multicolumn{2}{|c|}{142} & \multicolumn{2}{|c|}{60} & \multicolumn{2}{|c|}{52} & \multicolumn{2}{|c|}{24} & \multicolumn{2}{|c|}{615} \\
\hline $\begin{array}{l}\text { Población que se quedó en el país por año } \\
\text { (porcentaje) }\end{array}$ & \multicolumn{2}{|c|}{$21.91 \%$} & \multicolumn{2}{|c|}{$23.44 \%$} & \multicolumn{2}{|c|}{$23.74 \%$} & \multicolumn{2}{|c|}{$22.43 \%$} & \multicolumn{2}{|c|}{$71.26 \%$} \\
\hline Incremento acumulado anual & \multicolumn{2}{|c|}{142} & \multicolumn{2}{|c|}{202} & \multicolumn{2}{|c|}{254} & \multicolumn{2}{|c|}{278} & \multicolumn{2}{|c|}{893} \\
\hline
\end{tabular}

Tabla 3. Registro de ciudadanos venezolanos que cruzaron el PF de Lago Agrio entre 2013-2017 Fuente: Ministerio del Interior, 2018, adaptado por el autor.

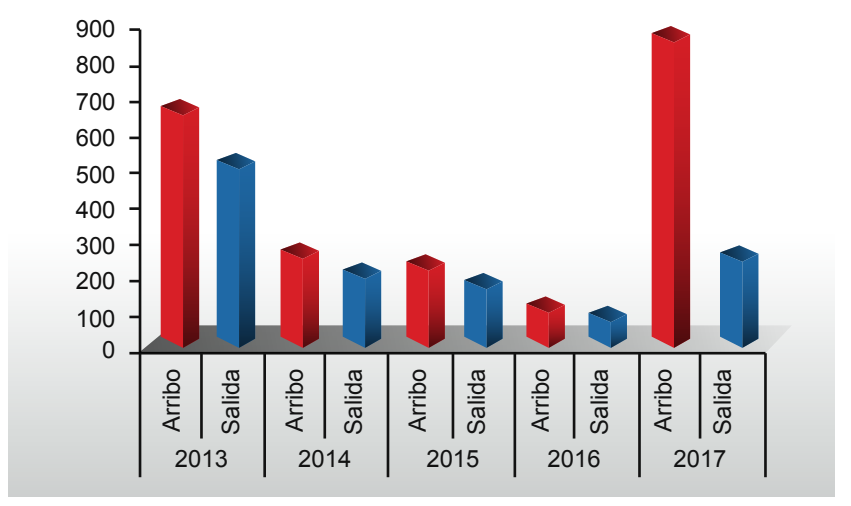

Figura 3. Relación entre arribo y salida de venezolanos por el PF de Lago Agrio (2013 y 2017)

Fuente: Ministerio del Interior, 2018, adaptado por el autor.

En la figura 4 se observa gráficamente el incremento anual acumulado de población que se quedó en el país entre 2013 y 2017, luego de cruzar el PF de Lago Agrio:

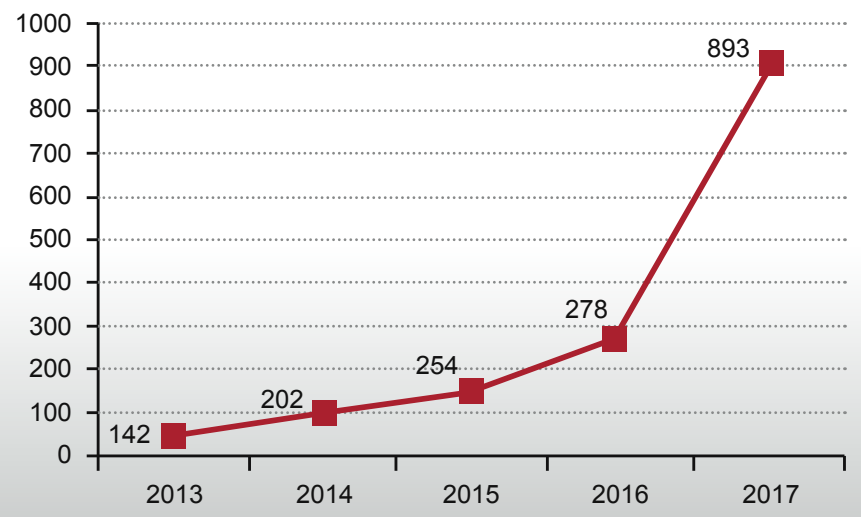

Figura 4. Incremento anual acumulado de venezolanos que se quedaron en Ecuador luego de ingresar por el PF Lago Agrio (2013 - 2017)

Fuente: Ministerio del Interior, 2018, adaptado por el autor. 
Es posible inferir que hasta el 31 de diciembre de 2017, 893 ciudadanos venezolanos se quedaron en el país luego de su ingreso por este PF (asumiendo que los mismos no han emigrado a otros países a través de pasos fronterizos no oficiales).

Solo en este año se presentó un incremento del $71.26 \%$ con respecto al año 2016 , lo cual está acorde al comportamiento de esta variable a nivel nacional. Es conveniente mencionar que, según la misma fuente, este es el lugar por donde entran más venezolanos empleando la vía terrestre, después del PF Rumichaca (provincia del Carchi).

Por otra parte, Colombia es otro país que aporta con un alto número de migrantes hacia el Ecuador, como efecto del conflicto interno que mantiene con grupos ilegales armados (GIA's), conformados por disidencias de las Fuerzas Armadas Revolucionarias de Colombia (FARC-EP), tras la firma del acuerdo de paz. Mencionados GIA's mantienen su capacidad para desestabilizar las condiciones de seguridad en ámbitos urbanos y rurales, dinamizar las economías criminales e influir negativamente en la implementación de los puntos de dicho acuerdo (Álvarez, Pardo y Cajías, 2018).

En efecto y según Álvarez et al. (2018), entre junio de 2016 y marzo de 2018, estos grupos cometieron 147 acciones delictivas en 13 departamentos colombianos y 8 en Ecuador, observándose un incremento en estos hechos tal como se demuestra en la figura 5:

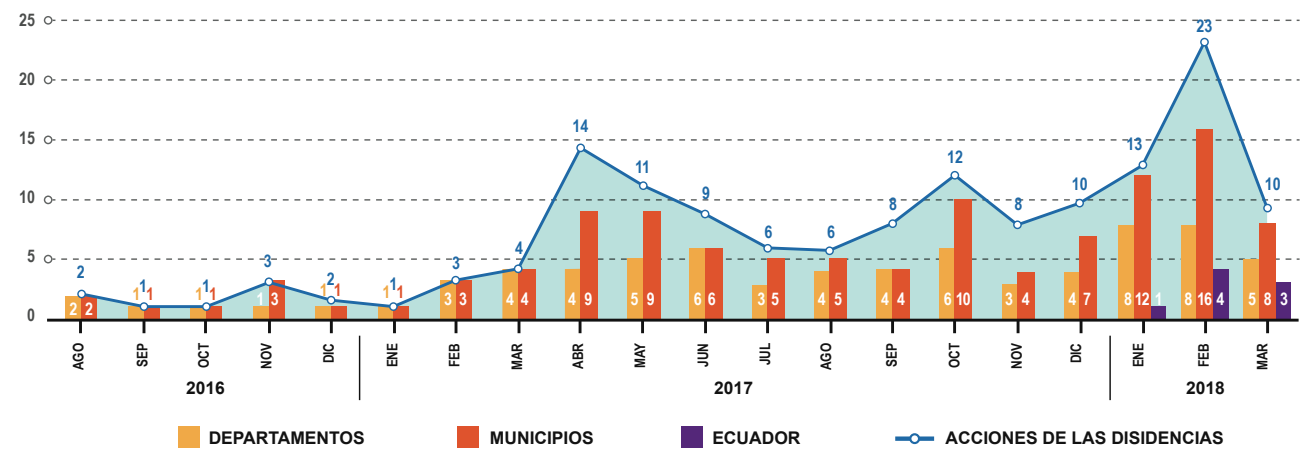

Figura 5. Acciones de las disidencias confirmadas en Colombia (junio 2016 - marzo 2018) Fuente: Álvarez et al., 2018

La intensificación de estas acciones en el territorio colombiano, también pueden visualizarse en mapas que las registran semestralmente, tal como se detalla en la figura 6 :
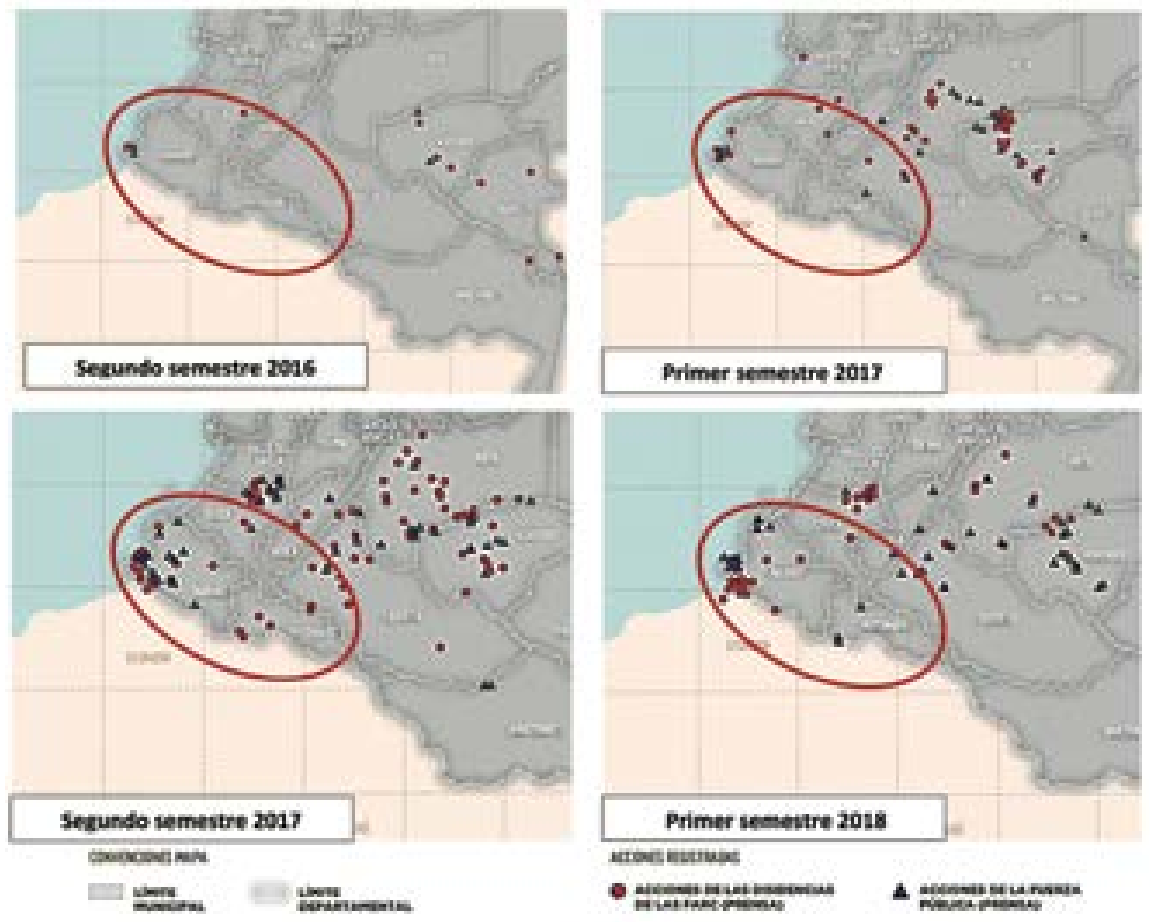

Figura 6. Ubicación de actos delictivos cometidos por disidencias en Colombia (junio 2016 - marzo 2018) Fuente: Álvarez et al., 2018 
Es posible verificar que departamentos fronterizos como Nariño y Putumayo registran un aumento sostenido del índice delictivo, con la correspondiente reducción en la seguridad colectiva de la zona y la generación de migración forzosa hacia Ecuador.
Complementariamente, la figura 7 muestra el nivel de incidencia y presencia de refugios temporales de los grupos irregulares armados en la frontera norte ecuatoriana durante el año 2016, según información compilada por el Instituto Geográfico Militar (I.G.M):

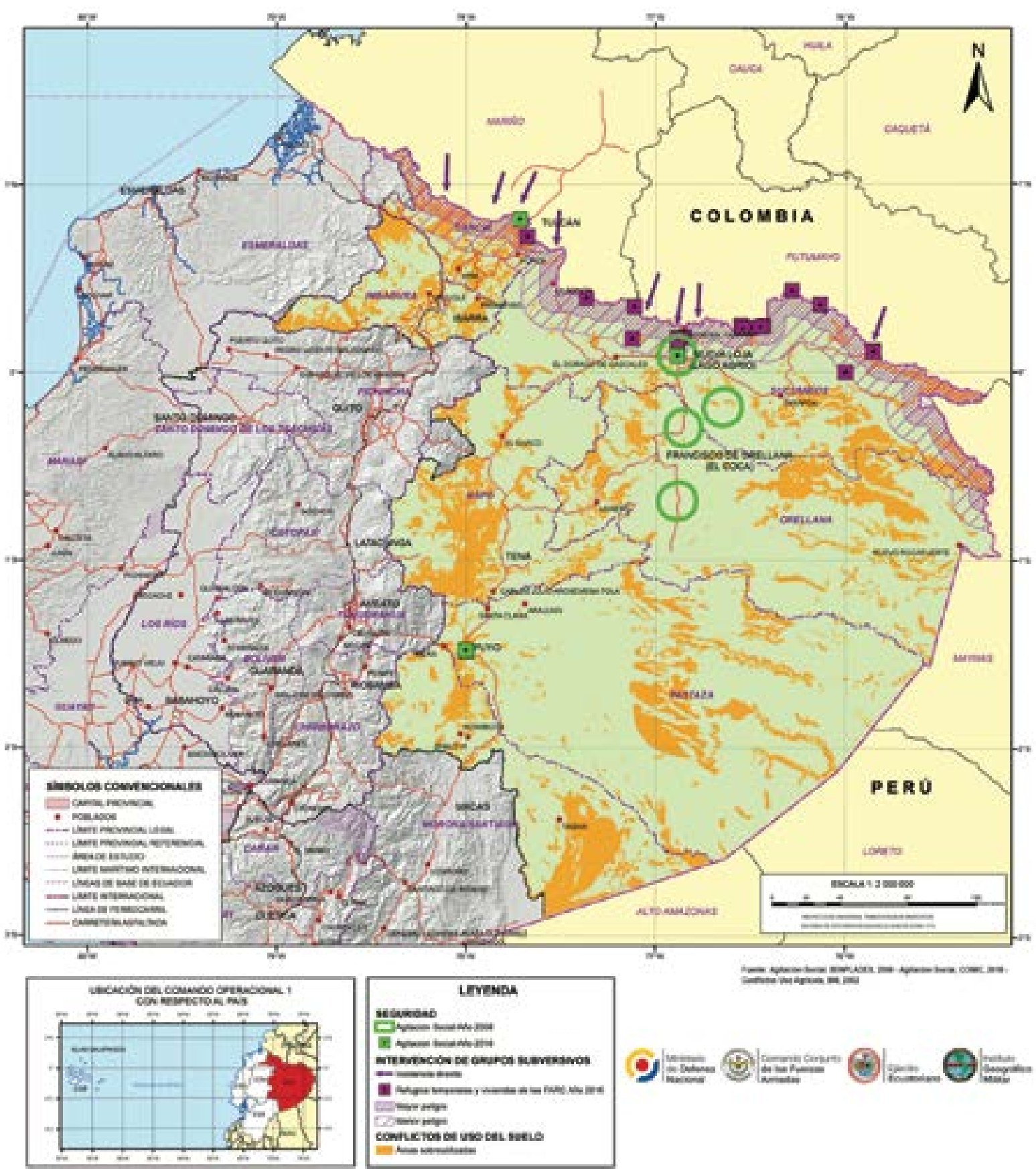

Figura 7. Incidencia de GIA’s en la frontera norte ecuatoriana durante el año 2016 Fuente: Instituto Geográfico Militar, 2017 
Según este mapa y en comparación con la figura 8 , el área considerada de mayor peligro está al interior de la Zona Fronteriza Terrestre (ZF) de 20 kilómetros. Es oportuno mencionar que la ZF está sujeta a restricciones de seguridad con presencia y vigilancia militar (Presidencia de la República del
Ecuador, 2007), lo cual habilita la ejecución continua de operaciones en el ámbito interno (OAI) por parte de las Fuerzas Armadas ecuatorianas para neutralizar potenciales amenazas y garantizar la estabilidad de la seguridad en la frontera norte.

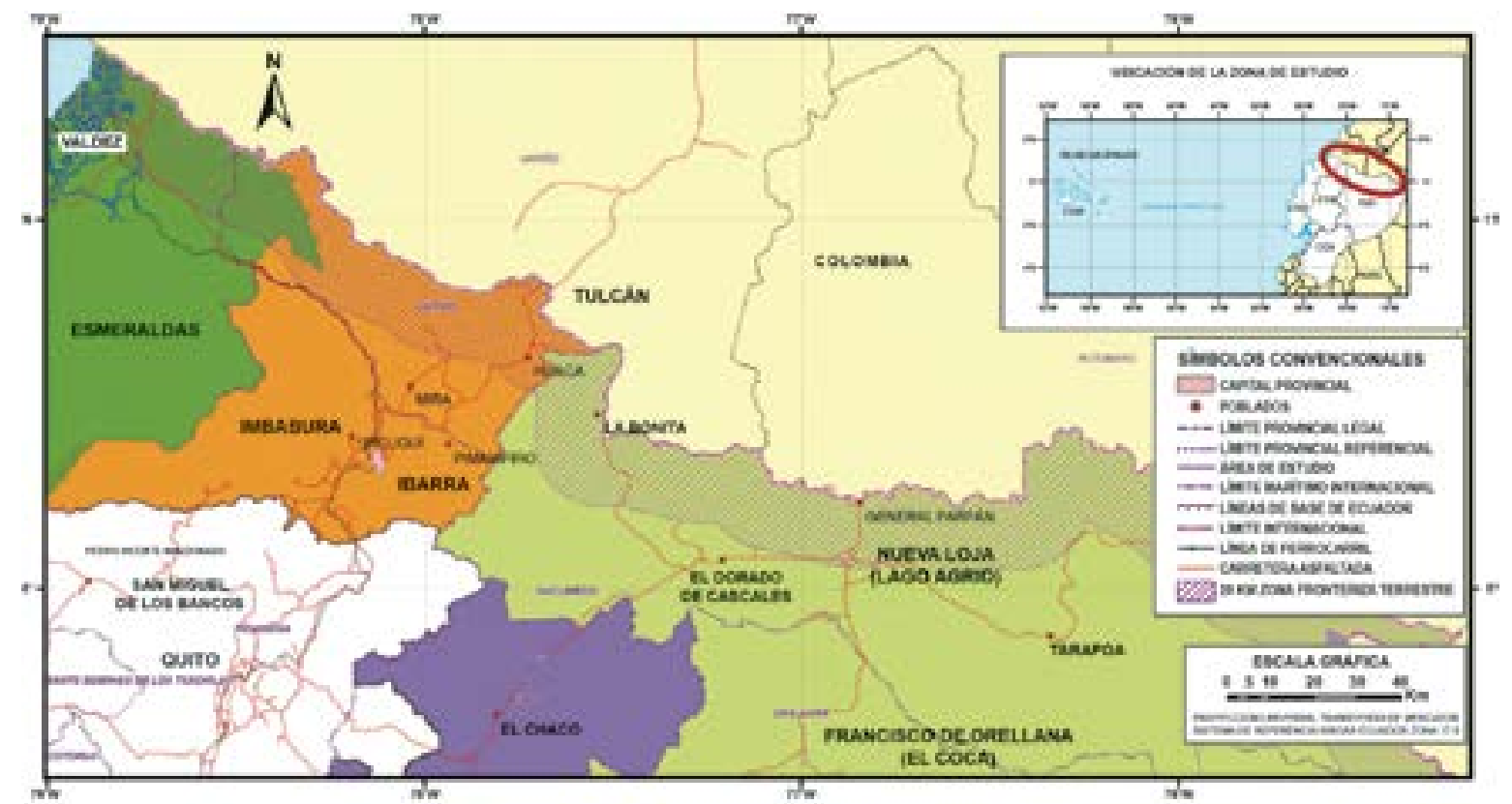

Figura 8. Zona Fronteriza (20 km) en el Límite Político Internacional con Colombia

Fuente: Instituto Geográfico Militar, 2018a

En adición a esta problemática, la Oficina de las Naciones Unidas contra la Droga y el Delito (UNODC), reportó un incremento de cultivos de coca a un ritmo del $45 \%$ anual desde el año 2013 en distintos lugares del territorio colombiano, pasando de 48.000 hectáreas en el año en referencia, a 146.000 hectáreas en el 2016. El año 2017 aportó un incremento adicional del 17\% hasta alcanzar una superficie de 171.000 hectáreas de este tipo de cultivos ilícitos (SIMCI-UNODC, 2018).

La figura 9 demuestra que dos de los tres departamentos colombianos con mayor cantidad de cultivos de coca son Nariño y Putumayo, precisamente aquellos territorios en los cuales existen disidencias de las FARC-EP, agudizando aún más el nivel de inseguridad social por efecto del narcotráfico y la violencia en la zona.

El crecimiento de la superficie cultivada con coca en zonas de frontera estaría asociado, no solo a una débil presencia institucional colombiana, sino también a condiciones geoestratégicas propicias para el desarrollo de la actividad ilegal (SIMCI-UNODC, 2018).

A pesar del emprendimiento de varios esfuerzos y reuniones entre el gobierno colombiano y autoridades

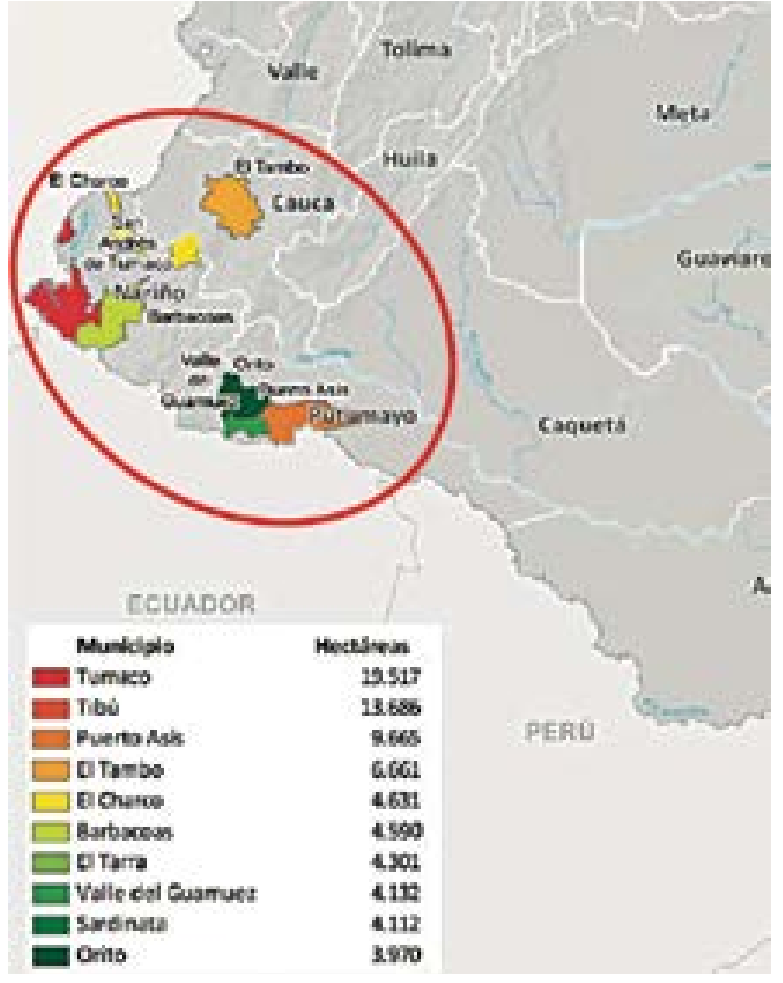

Figura 9. Municipios colombianos con mayores áreas sembradas de coca en 2017

Fuente: SIMCI-UNODC, 2018 
de Ecuador para fortalecer los mecanismos de intercambio de información y reforzar la vigilancia y la protección de las áreas fronterizas (Álvarez et al., 2018), no ha sido posible detener aún los desplazamientos poblacionales colombianos legales e ilegales hacia el interior del Ecuador en las provincias de Esmeraldas, Carchi y Sucumbíos, los cuales son permanentemente evidenciados por la ACNUR, por la OIM y por las entidades ecuatorianas responsables del control migratorio y de la seguridad pública ${ }^{1}$.

\section{IMPACTO EN LA SEGURIDAD INTERNA DEL ECUADOR}

La figura 10 demuestra el impacto de este escenario externo en la seguridad interna ${ }^{2}$ ecuatoriana. Se observa el cometimiento de actividades ilícitas en zonas cercanas a su frontera norte y a las vías de comunicación que conducen a ella. Entre algunas de las actividades referidas, en el periodo 2014 - 2016, se encuentran: tráfico de armas, municiones y explosivos, minería ilegal, tala ilegal de madera y plantaciones ilícitas (Instituto Geográfico Militar. 2017).

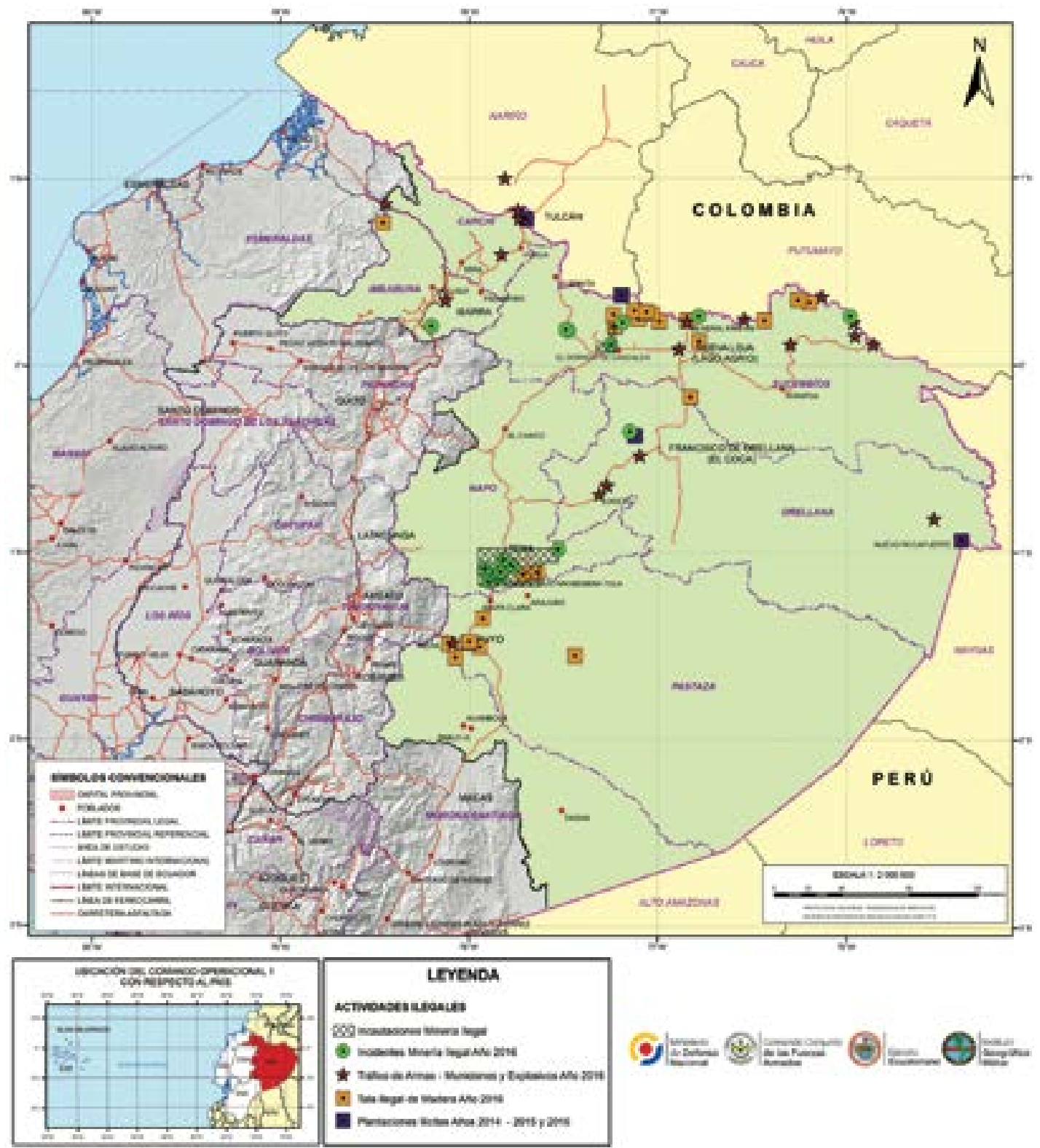

Figura 10. Mapa de actividades ilegales entre 2014 y 2016 Fuente: Instituto Geográfico Militar, 2017

1 Seguridad pública: control y mantenimiento del orden público frente a amenazas de alteración de ese orden, para lo cual el estado utiliza a la Fuerza Pública de manera progresiva, pudiendo utilizar los estados de excepción, ante un estado de grave conmoción interna (Academia de Guerra del Ejército, 2018).

2 Seguridad interna: comprende todas las medidas y acciones realizadas por el poder constituido para hacer frente, dentro de las fronteras del país, a las amenazas y factores de riesgo, de cualquier origen, naturaleza o forma (Academia de Guerra del Ejército, 2018). 
Es importante aclarar que el control fronterizo en esta zona se dificulta debido a la existencia de 43 pasos ilegales: 7 en Esmeraldas, 25 en Carchi y 11 en Sucumbíos (Instituto Geográfico Militar, 2018a), a través de los cuales, además de existir un flujo permanente de población migrante (cuyo destino final es incierto), circularían: contrabando, insumos para los GIA's, precursores para droga, etc. Estos pasos ilegales se detallan, de manera referencial, en la figura 11:
La provincia de Sucumbíos es una de las más afectadas. Su condición geográfica (fronteriza, con vegetación tropical, ampliamente rural, alejada geográficamente del centro político del Estado, de amplia extensión superficial, con disponibilidad de vías fluviales, terrestres y pasos ilegales fronterizos, entre otras características) podría derivar condiciones ideales para el cometimiento de actividades delictivas.

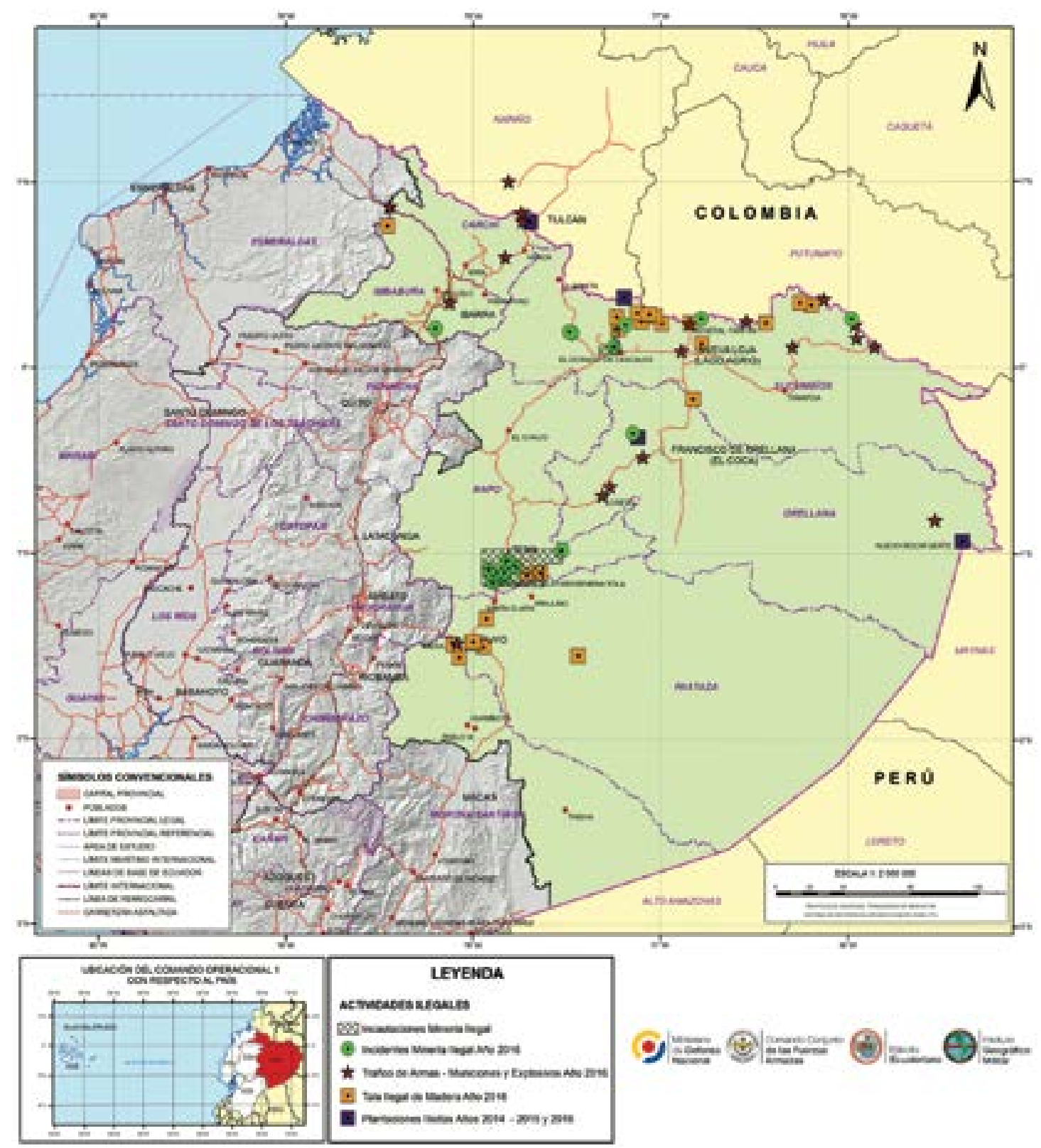

Figura 11. Mapa referencial de pasos ilegales en la frontera norte registrados en el 2018.

Fuente: Instituto Geográfico Militar, 2018a 
Según la FLACSO (2011), el asesinato es el delito que más se comete en esta provincia, el cual no se ajusta a una acción relacionada a delitos comunes, sino a ajustes de cuentas y narcotráfico. Las víctimas suelen ser parte de grupos irregulares armados colombianos que pasan a territorio ecuatoriano y son asesinados (FLACSO, 2011).

Con lo expuesto anteriormente, se evidencia el contemporáneo impacto del conflicto interno colombiano en todo el corredor fronterizo con el Ecuador, a pesar de los esfuerzos derivados del Acuerdo de Paz en el vecino país. Este impacto altera el nivel de seguridad colectiva ${ }^{3}$ y ocasiona desplazamientos poblacionales colombianos y ecuatorianos (y de migrantes de otros países que habitan en la zona), siendo el punto de partida para el aparecimiento de masas de población flotante en las provincias fronterizas y en el Ecuador en general. Este es precisamente el grupo de población al que se orienta este análisis.

\section{Masas de población migrante y flotante en el Ecuador: su vulnerabilidad}

La combinación entre este escenario de violencia e inseguridad, la frecuente vulnerabilidad socio-económica de este grupo poblacional y su alto nivel de nomadismo, podrían producir condiciones ideales para que ciudadanos extranjeros estén expuestos a sentirse atraídos al cometimiento de delitos de diferente índole, incrementando el nivel de inseguridad en general.

Según la Organización Internacional para las Migraciones de las Naciones Unidas (2018b), esta vulnerabilidad se evidencia en Ecuador con un $37 \%$ de venezolanos sufriendo discrimación y un $88 \%$ de aquellos que consiguieron trabajo, recibiendo un ingreso mensual menor a un salario básico. Las necesidades fundamentales identificadas en este segmento de población son: alimentación, generación de ingresos o empleo y asistencia de documentos.

La edad preponderante de estos migrantes oscila entre los 18 y 40 años. Además, similares circunstancias sacuden a los migrantes colombianos, sobre todo en localidades cercanas a la frontera norte.

A pesar de que el Estado cuenta con información oficial que permite inferir (al menos referencialmente) la cantidad de migrantes que permanecen en el país, no es posible mantener una base de datos oficial y continuamente actualizable de información de su ubicación cotidiana. Por lo tanto, y para enfocar cualquier estrategia de atención a sus problemas y garantizar la seguridad colectiva, es fundamental responder a varias preguntas claras: ¿dónde viven?, ¿cuáles son su principales actividades laborales?, ¿cuáles son sus necesidades prioritarias?, ¿qué porcentaje estaría o ha estado inmerso en actividades ilícitas?, ¿cuántos migrantes estárían erradicados en la Zona Fronteriza $(20 \mathrm{~km})$ de la provincia de Sucumbíos?, ¿cuál es el patrón de movimiento de esta masa poblacional?, etc. Esta caracterización es primordial.

Cabe mencionar que el último censo de población y vivienda, ejecutado por el Instituto Nacional de Estadísticas y Censos, data del año 2010. En aquel entonces las circunstancias detonantes de migración colombiana y venezolana eran direferentes a las actuales, lo cual disminuye el nivel de confianza en los datos oficiales relacionados a extranjeros residentes en la frontera norte en la actualidad.

En realidad, la migración irregular ${ }^{4}$ es un fenómeno social de naturaleza invisible $\mathrm{y}$, como tal, difícilmente podría ser caracterizado con precisión con las herramientas metodológicas convencionales (Texidó y Gurrieri, 2012)"publisher-place" : "Buenos Aires", "title" : "Panorama Migratorio de Amlu00e9rica del Sur", "type" : "book" \}, "uris" : [ "http://www.mendeley. com/documents/?uuid=d70ebc6b-0292-4f7b-b796302ed1d17fae" ] \} ], "mendeley" : \{ "formattedCitation" : "(Texidlu00f3 \& Gurrieri, 2012. Una forma de identificar el volumen de migrantes irregulares es a través de los procesos de regularización migratoria que ellos emprenden en el país de residencia, sin embargo no todos se regularizan por temor a ser deportados o ubicados por delincuentes colombianos. Otro indicador para medir el comportamiento de este fenómeno es el porcentaje de inadmitidos, detenidos y deportados por los países de destino, no obstante no todos son identificados y permanecen indefinidamente en el país.

En fin, es elemental describir, dimensionar, y modelar la dinámica de la población migratoria y su patrón de comportamiento para contextualizar integralmente sus problemas, como un punto de inicio al diseño e implementación de una solución sostenible en el ámbito de la seguridad.

3 Seguridad colectiva: percepción generalizada entre la población, de que existe un ambiente que garantiza el normal desarrollo de las actividades propias del convivir social (Academia de Guerra del Ejército, 2018).

4 Personas que se desplazan al margen de las normas de los Estados de envío, de tránsito o receptor. Desde el punto de vista del Estado receptor, el migrante no tiene la autorización necesaria ni los documentos requeridos por las autoridades de inmigración para ingresar, residir o trabajar en un determinado país. Desde el punto de vista de los países de envío la irregularidad se observa en los casos en que la persona atraviesa una frontera internacional sin documentos de viaje o pasaporte válido o no cumple con los requisitos administrativos exigidos para salir del país. 


\section{Acción del Estado en la seguridad interna en la Zona Fronteriza}

A partir del año 2010, tres han sido las líneas de política en cuanto a seguridad en las regiones fronterizas: a) en acciones económicas, el establecimiento de aranceles y de mecanismos de control ejecutados mediante aduanas y tributos; b) en defensa, garantizar la soberanía nacional y defensa territorial sobre la base de la militarización fronteriza y de la imposición de visas para extranjeros; y c) en seguridad pública, la preservación del orden público estatal a través del policiamiento para eliminar al enemigo interno (FLACSO, 2011).

No obstante, en el campo de la seguridad y defensa, y según Booth, Kestnbaum y Segal (2001), la organización militar debe enfrentar grandes cambios en cuanto a misiones, tamaño de fuerzas y estructura organizativa. Ello es reforzado por Malamud (2014) al identificar una redefinición en la estructura de valores y en la relación con la ciudadanía.

Por lo tanto el empleo militar como medida única para neutralizar el origen de la inseguridad interna en la Zona Fronteriza de la provincia de Sucumbíos, no es correcto. La relación entre la sociedad civil y la institución militar han evolucionado adaptándose a los dinámicos escenarios económicos y sociales en esta zona para mitigar a cada una de las amenazas existentes.

Este fenómeno social implica una flexibilización del paradigma que separó y caracterizó históricamente a estos sectores (Malamud, 2014), transformando al militar actual en un ente multifuncional y al civil en una fuente de apoyo mutuo y de coordinación interinstitucional.

Además, la creciente habilidad de las organizaciones delictivas para confundirse entre la población fronteriza, aumenta la dificultad para identificar la delgada línea que separa un estatus de riesgo de uno de normalidad, lo que impulsa el desarrollo de innovadoras y emergentes capacidades institucionales a un ritmo acelerado y proactivo para identificar y neutralizar oportunamente a los focos de inseguridad o inestabilidad.

La especificidad de cada una de estas amenazas requiere un manejo y respuesta especializada por parte de las Fuerzas Armadas, lo cual incluye una urgente profesionalización del militar en campos tradicionalmente ajenos a él (Martínez, 2017).
En tal virtud, la seguridad integral de la población y sus recursos depende de la habilidad que cada entidad estatal tenga para integrarse en una comunidad que mitigue oportunamente el riesgo ${ }^{5}$ de cada escenario. La inmediatez de la respuesta requerida deriva en una implícita cooperación multidimensional de todos los sectores, haciendo "flexible", no solo al militar, sino al Estado como tal.

En cuanto a las Fuerzas Armadas, estas operan de manera conjunta a través de Comandos Operacionales (C.O). La Zona Fronteriza en la provincia de Sucumbíos se encuentra bajo la jurisdicción del Comando Operacional No. 1 "NORTE". La planificación de las operaciones militares obedece al Proceso Militar en la Toma de Decisiones (PMTD). Este es un procedimiento continuo, dinámico y multidimensional para la solución de problemas militares y sirve al Comandante y a su Plana Mayor como herramienta para el desarrollo de apreciaciones, planes y órdenes. (Dirección de Doctrina, 2004). Como parte de este, la Preparación de Inteligencia en el Campo de Batalla (PICB), es un proceso continuo y sistemático de análisis del enemigo y del escenario en un área geográfica específica, que está diseñado para apoyar las apreciaciones y la toma de decisiones militares en una Plana o Estado Mayor (Comando de Educación y Doctrina del Ejército, 2015).

En operaciones en el ámbito interno, se requiere identificar a este "enemigo", entendiéndose como tal a todo riesgo potencial que ante la presencia de un factor detonante se transforme en amenaza para la seguridad. Una población migrante desatendida, vulnerable y con necesidades básicas altamente emergentes, podría convertirse en un riesgo. Su motivación de supervivencia, su incómoda condición laboral de migrante ilegal, la ausencia de fuentes de trabajo y la posibilidad de acceder a "dinero rápido" con el cometimiento de actividades ilícitas, crean un factor de riesgo que activaría una amenaza en una zona afectada por un alto índice delictivo.

Caracterizar a esta población (cantidad, ubicación, actividades, necesidades, desplazamientos, etc) es extremadamente esencial para el PICB como parte del PMTD. En efecto, los resultados de esta caracterización facilitarían la identificación proactiva y oportuna de áreas objetivo durante la planificación y posterior ejecución de OAI, mediante un análisis de la correlación que podría existir entre variables como: densidad poblacional migrante, ubicación de la misma, patrón de su desplazamiento, incidentes delictivos en la zona de estudio, vías de acceso empleadas para estos actos, pasos ilegales fronterizos, entre otros.

5 Situaciones y fenómenos latentes, de origen natural o antrópico, que podrían generar graves afectaciones a la seguridad pública y al Estado. Su posibilidad de ocurrencia es incierta. 
Debido al alto dinamismo de dicha población y del escenario en el que se desenvuelve, se requiere una herramienta que facilite el levantamiento continuo de información georreferenciada de dicho segmento poblacional, la registre espacialmente en sistemas de información geográfica (SIG), la procese analíticamente y permita visualizar los resultados por medio de una infraestructura de datos espaciales (IDE) ${ }^{6}$ (Suárez, Pindo y Balda, 2017), para proporcionar información de utilidad en la producción de inteligencia para la toma de decisiones en el ámbito militar.

\section{Caracterización espacial de la población migrante}

Para el caso en estudio, se postula una caracterización espacial de la población migrante como una alternativa basada en el diseño de una IDE orientada a facilitar los siguientes procesos:

1. Levantamiento de información de la población extranjera que habita en la Zona de Seguridad fronteriza en la provincia de Sucumbíos, mediante un censo de derecho ${ }^{7}$ ejecutado por el personal militar de la jurisdicción, empleando fichas censales impresas o tablets (dispositivos móviles) con aplicaciones como Open Data Kit - ODK ${ }^{8}$, desarrolladas para el efecto.

2. Seguimiento, registro, validación y almacenamiento la información obtenida para alcanzar una base de datos robusta y debidamente estructurada.

3. Procesamiento y caracterización espacial de la población migrante en la ZF de la provincia de Sucumbíos.

4. Análisis de la correlación entre variables definidas para el efecto (socio-económicas y de seguridad) con la finalidad de generar productos de utilidad para la producción de inteligencia en OAI.

5. Generación de un servicio web de mapas (WMS por sus siglas en inglés) para visualizar los productos cartográficos resultantes del análisis de correlación de variables, mediante el Geoportal del Instituto Geográfico Militar (I.G.M) u otro similar administrado por el Comando Operacional No. 1 "NORTE" con el apoyo y soporte técnico del I.G.M.

6. Evaluación del nivel de incremento de la eficiencia en la planificación y conducción de OAI en la
Zona Fronteriza de la provincia de Sucumbíos, como efecto de la caracterización de la población migrante en esta zona.

El método está diseñado para identificar y alcanzar la mejor caracterización posible de la población migrante en la zona. Como parte de la planificación para el censo deberá incluirse una fase de difusión coordinada con los organismos de comunicación social de cada uno los Gobiernos Autónomos Descentralizados y demás autoridades competentes. Se aprovecharía el desarrollo de las operaciones militares que ejecuten las unidades operacionales para ingresar a lugares alejados o de difícil acceso. Finalmente, se ejecutará el monitoreo permanente del avance y cubertura de las áreas planificadas para este proyecto.

No obstante estas coordinaciones, se debe reconocer que las principales limitantes estarán relacionadas a la distancia y acceso a áreas rurales sin vías de comunicación. Otra limitante sería el temor de la población a ciertas represalias que los grupos delictivos podrían tener por la colaboración con las fuerzas militares ecuatorianas. A pesar de ello, y considerando que actualmente no existe una base de datos de similares condiciones a las que se busca crear, el proyecto es de alto interés, demanda y viabilidad ${ }^{9}$.

\section{Conclusiones}

La contribución del conocimiento geográfico en diversas actividades encaminadas a la Seguridad y Defensa del país es cada vez más importante, especialmente en el ámbito de la coordinación y apoyo a las operaciones militares y en todos los niveles de conducción (Instituto Geográfico Militar, 2018b). La planificación y desarrollo de las mismas, requieren información geográfica específica de cuya confiabilidad y oportunidad dependerá el éxito o fracaso de una operación (Gutiérrez, 2015). En efecto, la captura, proceso y almacenamiento de la información obtenida con los SIG, brindan la posibilidad de realizar un producto de inteligencia en óptimas condiciones y con la mayor precisión posible, lo cual será determinante en la toma de decisiones (Radke, 2014).

6 IDE: conjunto de tecnologías, políticas, estándares y recursos humanos para adquirir, procesar, almacenar, visualizar, distribuir y potenciar el empleo de la información geográfica.

7 Se desarrolla en un período comprendido entre varios días hasta un par de meses dependiendo de la planificación. Se considera el registro de las personas que residen habitualmente en el hogar, aunque no se encuentren presentes cuando lleguen los encuestadores. Una de las preguntas que formulan los encuestadores es “¿dónde reside habitualmente?”. La finalidad básica es determinar la residencia habitual.

8 ODK: conjunto de herramientas libres y de código abierto que permite implementar soluciones para la recolección de datos usando equipos móviles con localización e imágenes.

9 En el Comando Operacional No. 1 "NORTE" (específicamente en la provincia de Sucumbíos y entre los años 2009 y 2011) se desarrolló el proyecto "Censo de Predios en la frontera norte" dentro de la ZF. Se alcanzaron resultados de gran utilidad para la producción de inteligencia. 


\section{Recomendaciones}

Sobre la base del análisis realizado, es recomendable explotar continuamente las capacidades y experiencia disponibles tanto en las unidades militares del C.O 1 "NORTE" como en el Instituto Geográfico Militar, con la finalidad de incrementar la eficiencia y eficacia de las operaciones en el ámbito interno para garantizar la seguridad interna en la zona fronteriza de la Provincia de Sucumbíos, a través de la caracterización espacial de la población migrante presente en esta zona.

\section{REFERENCIAS}

[7] Academia de Guerra del Ejército. (2018). Propuesta de Actualización de Manual de Empleo en las Operaciones en el Ámbito Interno. Quito.

[8] Álvarez, E., Pardo, D., \& Cajías, A. (2018). Trayectorias y dinámicas territoriales de las de las FARC. Bogotá.

[9] Asamblea Constituyente. (2008). Constitución de la República del Ecuador.

[10] Asamblea General de las Naciones Unidas. (1954). Convención sobre el Estatuto de los Refugiados. Ginebra. Retrieved from https://www. acnur.org/fileadmin/Documentos/ BDL/2001/0005.pdf?file=fileadmin/ Documentos/BDL/2001/0005

[11] Castles, S., \& Miller, M. (2004). La Era de la Migración. México. Retrieved from http://biblioteca. diputados.gob.mx/janium/bv/ce/scpd/ LIX/era_mig.pdf

[12] Comando de Educación y Doctrina del Ejército. (2015). Manual de Conduccion Militar. Quito.

[13] FLACSO. (2011). Gobernanza de la Seguridad Ciudadana en la Frontera Norte de Ecuador. FRONTERAS. Quito.

[14] Gutiérrez, J. (2015). Sistemas de Información Geográfico Militar. Buenos Aires. Retrieved from http:// cartomap.cl/escuela_militar/Sig/SIG04.pdf

[15] Instituto Geográfico Militar. (2017). Diagnóstico Cartográfico-Geográfico (Geoespacial) de la Frontera Ecuatoriana (Colombia-Perú). Quito.
[16] Instituto Geográfico Militar. (2018a). Atlas Cartográfico Geográfico Frontera Norte Ecuatoriana. Quito.

[17] Instituto Geográfico Militar. (2018b) Guía Metodológica para el Análisis de los Aspectos Militares del Terreno Mediante Sistemas de Información Geográfica (Primera Ed). Quito.

[18] Malamud, M. (2014). El nuevo “militar flexible." Revista Mexicana de Sociología, 4, 639-663. Retrieved from http://www.scielo.org.mx/pdf/ rms/v76n4/v76n4a5.pdf

[19] Ministerio del Interior. (2018). Flujo de venezolanos por unidad de control y tipo de residencia. Quito. Retrieved from https://www.ministeriointerior. gob.ec/wp-content/uploads/2018/02/ FLUJO-DE-VENEZOLANOS-20132017-POR-PUERTOS.pdf

[20] Organización Internacional para las Migraciones - OIM. (2018). Los términos clave de migración. Washington, Estados Unidos: OIM. Recuperado de: http://www.iom.int/es/ los-terminos-clave-de-migracion

[21] Presidencia de la República del Ecuador. (2007). Decreto Presidencial No. 433 - Áreas Reservadas del Ecuador. Quito.

[22] Radke, Y. (2014). Sistemas de información geográfica y su uso en unidades de inteligencia. Buenos Aires: Instituto de Inteligencia Militar de las Fuerzas Armadas Argentinas. Retrieved from https://www.academia. edu/29180655/Sistemas_de informacion_geografico_y_su_uso_ en_el_ambito_militar
[23] SIMCI-UNODC. (2018). Colombia Informe de Monitoreo de Territorios Afectados por Cultivos Ilícitos 2017. Bogotá.

[24] Suárez, S., Pindo, J., Balda, R. (2017). Implementación de plataforma para generación de encuestas georeferenciadas y visualización en una infraestructura de datos espaciales (IDE). Geoespacial-ESPE, 14(11), 106-115.

[25] Texidó, E. (OIM), \& Gurrieri, J. (2012). Panorama Migratorio de América del Sur. Buenos Aires: Organización Internacional para las migraciones. Retrieved from https://repository.oim.org.co/ bitstream/handle/20.500.11788/1399/ ROBUE-OIM 006. pdf? sequence $=1$ \&isAllowed $=\mathrm{y}$

[26] Zurita, E., González, M., \& Dávalos, E. (2017). Impacto de la Inmigración sobre el Crecimiento de la Renta Per Cápita de Ecuador (2001-2015). INNOVA Research Journal, 2(10), 147-161. Retrieved from file:///C:/ Users/torres/Downloads/Dialnet-Impa ctoDeLaInmigracionSobreElCrecimie ntoDeLaRentaP-6183859.pdf 